Alberta-Thy-32-94

gr-qc/9411001

October 1994

\title{
Action and Hamiltonian for eternal black holes
}

\author{
Valeri Frolov and Erik A. Martinez * \\ Theoretical Physics Institute, \\ University of Alberta, \\ Edmonton, Alberta T6G 2J1, Canada.
}

\begin{abstract}
We present the Hamiltonian, quasilocal energy, and angular momentum for a spacetime region spatially bounded by two timelike surfaces. The results are appplied to the particular case of a spacetime representing an eternal black hole. It is shown that in the case when the boundaries are located in two different wedges of the Kruskal diagram, the Hamiltonian is of the form $H=H_{+}-H_{-}$, where $H_{+}$and $H_{-}$are the Hamiltonian functions for the right and left wedges respectively. The application of the obtained results to the thermofield dynamics description of quantum effects in black holes is briefly discussed.
\end{abstract}

PACS numbers: 04.20.Cv, 05.30.Ch, 97.60.Lf

Typeset using REVTEX

*electronic addresses: frolov@phys.ualberta.ca, martinez@phys.ualberta.ca 


\section{INTRODUCTION}

One of the most important problems of black hole physics concerns the dynamical origin of black hole entropy and, more generally, the statistical mechanical foundations of black hole thermodynamics. A natural way to calculate the thermodynamical parameters of a black hole is to use a "complexification" approach [1] 3. However, in this framework there is simply no room for the internal degrees of freedom of the black hole since one deals explicitly only with the Euclidean version of the black hole exterior. That is why it is important to begin with the usual Lorentzian description of a black hole in order to understand the dynamical origin of its internal degrees of freedom.

In order to describe a state of a black hole in the Lorentzian approach one uses a spatial slice which crosses the horizon. In the general case such a slice crosses also a singularity inside the black hole horizon so that fixing a state requires additional data describing the singularity. This difficulty can be overcome by considering the 'eternal version' of a black hole, as done recently in [4]. The main idea of this approach is as follows: a black hole which is formed as a result of gravitational collapse is described at late time by a stationary metric. One can use analytical continuation to connect the late time geometry of this configuration with the spacetime of an eternal black hole possessing the same parameters (mass $M$, angular momentum $J$, charge $Q$, etc.). This analytically continued spacetime is called the 'eternal version' of the original black hole. The Penrose diagram for such a spacetime is shown in Figure 1. Classical or quantum excitations of the black hole at late time can be traced back in the spacetime of its 'eternal version' to some initial global Cauchy surface. For example, one can choose a slice $\Sigma$ representing a spatial section at the moment of time symmetry as such a surface. The surface $\Sigma$ is an Eintein-Rosen bridge with wormhole topology $R^{1} \times S^{2}$ (Figure 2). In the general case when the black hole is not static

and possesses external as well as internal excitations this construction allows one to relate the excitations of a real black hole at late time with the corresponding field perturbations at $\Sigma$. The gravitational field perturbations on $\Sigma$ can be identified with small deformations 
of the three-geometry of the Einstein-Rosen bridge. This construction can be generalized to the situation when the perturbations are not small. In this case the initial data on $\Sigma$ can be described as (not necessarily small) deformations of the Einstein-Rosen bridge plus initial data for the non-gravitational degrees of freedom on the distorted Einstein-Rosen bridge provided the constraint equations are satisfied. In the framework of this construction one may consider, instead of different states of a real black hole at late time, different states of the corresponding Einstein-Rosen bridge (or the corresponding deformations of it). The latter problem appears to be much simpler analytically. In particular, it allows a simple description of the internal degrees of freedom of a black hole 四. The bifurcation surface of the Killing horizon is a two-dimensional surface of minimal area on $\Sigma$ which divides the surface $\Sigma$ in two parts denoted by $\Sigma_{+}$and $\Sigma_{-}$. The initial data at $\Sigma_{-}$naturally define the internal degrees of freedom of a black hole.

A black hole considered as a part of a thermodynamical system can be in equilibrium with surrounding thermal radiation provided the temperature of the radiation (measured at infinity) coincides with the black hole temperature. This equilibrium is stable only if the black hole is contained inside a cavity of finite size where the boundary data of the appropriate statistical ensemble are specified. (For example, for a non-rotating black hole inside a spherical boundary of radius $r_{0}$ with fixed temperature $T_{0}$, stable equilibrium requires $r_{0} \leq 3 M$.) The important role played by boundaries in formulations of black hole thermodynamics has been stressed by York and collaborators [2,3].

An interesting attempt to develop thermodynamical formulations of black holes directly in the Lorentzian approach was made in Ref. [5]. However, as indicated above, the existence of singularities in the black hole interior creates potential difficulties in such an approach. That is the reason why it is interesting to develop the Lorentzian approach beginning directly with the eternal version of a black hole [6]. However, a price to be paid is that a single external two-dimensional boundary does not restrict anymore a finite three-dimensional volume on a spacelike slice with wormhole topology. In order to analyze problems involving a finite volume for such spaces one needs to consider two (holomorphic) surfaces, each 
one with two-sphere topology. The main aim of this paper is to generalize the results of Ref. [7] to the case of eternal black holes and spacetime regions contained between two timelike boundaries. Namely, we construct the gravitational Hamiltonian and generalize the quasilocal energy and angular momentum definitions for this kind of situations. Special attention is paid to the case when the external boundary is located, as usual, in the black hole exterior while the other boundary (the internal one) is located either at the external part $\Sigma_{+}$or at the internal part $\Sigma_{-}$of the Einstein-Rosen bridge.

The construction of Hamiltonian and quasilocal energy for a spatially bounded region of an eternal version of a black hole is useful when considering quantum properties of black holes. Recently a no-boundary ansatz (analogous to the Hartle-Hawking no-boundary ansatz in quantum cosmology) has been proposed to define a wave function of a black hole [四]. Such a wave function is a functional of the configuration space of deformations of the EinsteinRosen bridge. For field perturbations this wave function describes a Hartle-Hawking state of linear fields propagating in the background of a black hole. Restriction to a part of the system (either internal or external) generates a thermal density matrix. The thermal nature of the density matrix is directly related with the special structure of the Hamiltonian $H$ for small perturbations, which is of the form $H=H_{+}-H_{-}$, where $H_{+}$and $H_{-}$are the Hamiltonians for perturbations on the external and internal parts of an Einstein-Rosen bridge respectively. Israel [8] has noted that this situation is in complete accord with the thermofield dynamics approach to thermodynamics developed in Ref. [9]. The results obtained in this paper allow one in principle to develop this analogy beyond perturbations to the strong gravity regime, in which all the quantities, and in particular the Hamiltonian, refer to the gravitational field of the black hole itself. The total Hamiltonian for the system is encoded in the boundary conditions and posseses the general form $H=H_{+}-H_{-}$, where $H_{+}$and $H_{-}$are the gravitational Hamiltonians for the external and internal regions of a distorted black hole. A full generalization of the thermofield dynamics approach to black holes can be related therefore with the study of geometrodynamics of an 'eternal version' of a black hole. 
The paper is organized as follows. We begin by discussing in Section II the geometry of the spacetime of a Schwarzschild-Kruskal eternal black hole and its natural foliation connected with the global Killing vector field $\xi_{t}$ ("tilted foliation"). We describe generalizations of the "tilted foliations" for a general spacetime (without a Killing vector) and general kinematical relations applicable to "tilted" as well as to the standard ("untilted") foliations. The general expressions for Hamiltonian, quasilocal energy and angular momentum are obtained by using a "tilted foliation" in Sections III, IV, and V respectively. The dynamics of the standard ("untilted") foliation is discussed in Section VI. The possible relation of the obtained results with the thermofield dynamics approach to black hole thermodynamics is discussed in Section VII. We use the sign conventions of Ref. [10], and units are chosen so that $G=c=\hbar=1$.

\section{FOLIATIONS AND KINEMATICS}

\section{A. Topology}

Before considering the more general case of a distorted black hole, we describe some properties of stationary vacuum eternal black hole solutions. Consider at first the case of a static, non-rotating black hole. The corresponding Schwarzschild-Kruskal line element reads:

$$
d s^{2}=\frac{-32 \mathcal{M}^{3}}{r} e^{-r / 2 \mathcal{M}} d U d V+r^{2} d \Omega^{2},
$$

where $\mathcal{M}$ represents the Arnowitt-Deser-Misner (ADM) mass of the static black hole [11, $d \Omega^{2}$ is the line element of the unit two-sphere, and the radial coordinate is regarded as a function of the ingoing and outgoing null coordinates $(U, V)$ as

$$
(1-r / 2 \mathcal{M}) e^{r / 2 \mathcal{M}}=U V
$$

The corresponding Penrose diagram is shown in Figure 1. The Schwarzschild-Kruskal spacetime [10] is the union of four regions (wedges) $R_{+}, R_{-}, T_{+}$, and $T_{-}$. The regions $R_{+}$and 
$R_{-}$are asymptotically flat. In the $R_{+}$region, the Kruskal coordinates $(U, V)$ satisfy $U<0$, $V>0$, while in $R_{-}, U>0, V<0$. The Killing vector field $\xi_{t}$ connected with the time symmetry is

$$
\xi_{t}^{\mu} \partial_{\mu}=\frac{1}{4 \mathcal{M}}\left(V \partial_{V}-U \partial_{U}\right)
$$

This Killing vector is timelike in $R_{ \pm}$, spacelike in $T_{ \pm}$, and becomes null at the future $\left(H^{+}\right)$ and past $\left(H^{-}\right)$horizons. It is future oriented in $R_{+}$and past oriented in $R_{-}$, whereas it vanishes at the bifurcation surface $\tilde{S}_{0}=H^{+} \cap H^{-}$.

Surfaces orthogonal to $\xi_{t}$ are defined by the equation $t=$ constant, where the scalar function $t$ is defined as

$$
t=2 \mathcal{M} \ln \left|\frac{-V}{U}\right|
$$

The line element (2.1) is invariant with respect to the discrete symmetries

$$
\begin{gathered}
\mathbf{I}: U \rightarrow-U ; V \rightarrow-V, \\
\mathbf{T}: U \rightarrow-V ; V \rightarrow-U .
\end{gathered}
$$

The surface $\tilde{\Sigma}$ is defined by the equations $t=0$ or, equivalently, $U+V=0$. This surface is invariant under $T$-reflections and, (as well as any other surface $t=$ constant) has EinsteinRosen bridge topology $R^{1} \times S^{2}$ (Figure 2). We denote by $\tilde{\Sigma}_{ \pm}$the parts of $\tilde{\Sigma}$ lying in $R_{ \pm}$ respectively. The line element (2.1) restricted to the surface $\tilde{\Sigma}$ reads

$$
d l^{2}=d y^{2}+r^{2}(y) d \Omega^{2}
$$

where the quantity $y$, defined by

$$
d y= \pm \frac{d r}{\sqrt{1-2 \mathcal{M} / r}}
$$

represents the proper geodesic distance from the "throat" of the bridge located at $r=r(y=$ $0)=r_{+}=2 \mathcal{M}$. We choose $y$ to be positive in $\tilde{\Sigma}_{+}$and negative in $\tilde{\Sigma}_{-}$. The set $(t, y, \theta, \phi)$ can be used as canonical coordinates everywhere in $R_{ \pm}$outside the surface $\tilde{S}_{0}$. These coordinates are right-oriented in $R_{+}$and left-oriented in $R_{-}$. 
The second homotopy group of $\Sigma \equiv R^{1} \times S^{2}$ is $\pi_{2}\left(R^{1} \times S^{2}\right)=\mathbf{Z}$. This means that, besides the two-dimensional topologically spherical surfaces in $\tilde{\Sigma}$ which can be contracted to a point, there also exist in $\tilde{\Sigma}$ non-contractible two-dimensional spheres. An example of such a sphere is the bifurcation surface $\tilde{S}_{0}$ or any other surface obtained from it by continuous transformations. Therefore, in order to restrict any finite three-dimensional volume on the $t=$ const. hypersurface it is necessary to have as boundaries two such topological spheres $\left(\tilde{S}_{+}\right.$and $\left.\tilde{S}_{-}\right)$. In the particular case when the boundary surfaces are round spheres, they are defined by the equations $y=y_{+}$and $y=y_{-}$. The spatial region restricted by these spheres has topology $I \times S^{2}$, where $I$ is a finite interval. In mathematical literature, this region is known as cobordism between the boundary surfaces $\tilde{S}_{+}$and $\tilde{S}_{-}$.

A natural way of describing dynamics of fields propagating on the eternal black hole background is to use the Killing time $t$ as the evolution parameter. Two different physical situations are possible in principle: (1) both boundary surfaces are located in the same region of the spacetime (either $R_{+}$or $R_{-}$), and (2) the boundary surfaces are located in different regions. In case $(1)$, the $t=$ const. slices do not intersect each other, so that the foliation is of the standard form. In case (2), two different slices $\tilde{\Sigma}^{\prime}\left(t=t^{\prime}\right)$ and $\tilde{\Sigma}^{\prime \prime}\left(t=t^{\prime \prime}\right)$ intersect at the two-dimensional bifurcation sphere $\tilde{S}_{0}$. We call the sequence of slices $\tilde{\Sigma}_{t}$ (defined by the equation $t=$ constant, with $\left.t^{\prime} \leq t \leq t^{\prime \prime}\right)$ which intersect at the same bifurcation surface $\tilde{S}_{0}$ the "tilted foliation". For a "tilted foliation" the spacetime domain $M$ lying between $\tilde{\Sigma}^{\prime}$ and $\tilde{\Sigma}^{\prime \prime}$ consists of two wedges $M_{+}$and $M_{-}$located in the right $\left(R_{+}\right)$and left $\left(R_{-}\right)$sectors of the Kruskal diagram. In both cases, if we restrict ourselves by considering the field dynamics in a finite region of spacetime restricted by boundary slices $\tilde{\Sigma}^{\prime}$ and $\tilde{\Sigma}^{\prime \prime}$, we need also to specify boundary conditions at two timelike boundaries, which we denote by $\tilde{B}_{ \pm}$. Each of these boundaries is the result of time evolution from $t^{\prime}$ to $t^{\prime \prime}$ of a topological sphere homotopic to $\tilde{S}_{0}$. Both type of foliations mentioned above can be defined in the more general case when one does not restrict oneself by considering the dynamics with respect to the Killing time $t$.

We describe at first the construction of the "tilted" and "untilted" foliations for the Kerr-Newman spacetime. In the case of a rotating black hole, the Killing vector $\xi_{t}$ (uniquely 
defined as the Killing vector which is timelike at spatial infinity) is not hypersurface orthogonal anymore. Nevertheless, its linear combination with the Killing vector $\xi_{\phi}$ (corresponding to axial symmetry)

$$
u^{\mu}=A\left(\xi_{t}^{\mu}+\omega \xi_{\phi}^{\mu}\right)
$$

defines a unit vector $u^{\mu}$ normal to a hypersurface $\Sigma_{t}$, provided that

$$
\omega=-\frac{g_{t \phi}}{g_{\phi \phi}} ; A=\sqrt{\frac{g_{\phi \phi}}{g_{t \phi}^{2}-g_{t t} g_{\phi \phi}}} .
$$

Here $g_{\mu \nu}$ denotes the coefficients of the Kerr-Newman metric in the standard Boyer-Lindquist coordinates [10]. The hypersurface $\Sigma_{t}$ is defined by the equation $t=$ constant. It is easy to verify that $\xi_{\phi} \cdot u=0$, so that the $u$-observers are zero angular momentum observers [12].

The topology of the surface $t=0$ (as well as any other surface $t=$ constant) in the KerrNewman spacetime is the same as the topology of the $t=0$ slice for a Schwarzschild-Kruskal black hole, namely, $R^{1} \times S^{2}$. The $t=$ constant slices cross each other at a bifurcation surface $\tilde{S}_{0}$ with the topology of a two-sphere. The hypersurfaces $\Sigma_{t}$ form a "tilted foliation" for the Kerr-Newman spacetime. The "untilted" foliation is generalized in a similar way.

The constructions of both "tilted" and "untilted" foliations can be easily generalized to the case of a distorted black hole. Let us consider a three-dimensional surface, denoted by $\Sigma$, with Eintein-Rosen bridge topology $\left(R^{1} \times S^{2}\right)$ and whose three-geometry is such that the corresponding (distorted) Einstein-Rosen bridge connects two asymptotically flat spaces. We choose this three-geometry and its time derivatives in such a way that the gravitational constraint equations are satisfied. These initial data can be used to determine the subsequent spacetime evolution by using the vacuum Einstein equations. We assume that this evolution defines a regular spacetime in some domain $M$ lying both to the future and to the past of the initial Cauchy surface $\Sigma$. We also assume that it is possible to choose two different spacelike Cauchy surfaces $\Sigma^{\prime}$ and $\Sigma^{\prime \prime}$ in this domain which intersect each other at the two-dimensional, topologically spherical spacelike surface $S_{0}$. Consider a differential one-to-one map $\Psi$ of the domain $M$ into the Schwarzschild-Kruskal spacetime possessing the folowing properties: 


$$
\Psi\left(\Sigma^{\prime}\right)=\tilde{\Sigma}^{\prime} ; \Psi\left(\Sigma^{\prime \prime}\right)=\tilde{\Sigma}^{\prime \prime} ; \Psi\left(B_{ \pm}\right)=\tilde{B}_{ \pm} ; \Psi\left(S_{0}\right)=\tilde{S}_{0} ; \Psi\left(S_{ \pm}\right)=\tilde{S}_{ \pm}
$$

This map transforms the region $A$ lying between $\Sigma^{\prime}$ and $\Sigma^{\prime \prime}$ into the region $\tilde{A}$ lying between $\tilde{\Sigma}^{\prime}$ and $\tilde{\Sigma}^{\prime \prime}$. We can use this map to provide foliations for the distorted black hole spacetime. These foliations are obtained by simply transfering the foliations of the SchwarzschildKruskal spacetime by the map $\Psi$ onto $M$. Certainly there exist an infinite set of maps $\Psi$ that accomplish this, and one can choose anyone of them. For a chosen map $\Psi$ the canonical coordinates $(t, y, \theta, \phi)$ introduced above for the "tilted foliation" of the Schwarzschild-Kruskal spacetime can be transfered from $\tilde{A}$ to the spacetime region $A$ on $M$. The coordinates $(t, y, \theta, \phi)$ are right-oriented in $R_{+}$and left oriented in $R_{-}$.

\section{B. Kinematics}

We discuss now the kinematics of a general spacetime $M$ whose line element has the form

$$
d s^{2}=-N^{2} d t^{2}+h_{i j}\left(d x^{i}+V^{i} d t\right)\left(d x^{j}+V^{j} d t\right)
$$

where $t$ is the time coordinate connected with the chosen foliation, and $N$ the corresponding lapse function. The four-velocity is defined by $u_{\mu}=-N \partial_{\mu} t$, and the lapse function $N$ is defined so that $u \cdot u=-1$. The four-velocity $u^{\mu}$ is the timelike unit vector normal to the spacelike hypersurfaces $(t=$ constant $)$ denoted by $\Sigma_{t}$. The vector $t^{\mu}$ that connects points with the same spatial coordinates in different slices is related to the four-velocity $u^{\mu}$ by

$$
t^{\mu}=N u^{\mu}+V^{\mu},
$$

so that $V^{i}=h_{0}^{i}=-N u^{i}$ is the shift vector. We choose the spacelike boundaries to coincide with surfaces of fixed values of $t$, so that the time coordinate $t$ is the scalar function that uniquely labels the foliation. We assume that the surfaces $\Sigma^{\prime} \equiv \Sigma_{t^{\prime}}$, and $\Sigma^{\prime \prime} \equiv \Sigma_{t^{\prime \prime}}$ are the spacelike boundaries of the spacetime region under consideration.

We assume that a three-dimensional timelike boundary $B$ consists of two disconnected parts $B_{+}$and $B_{-}: B=B_{+} \cup B_{-}$. The spacelike normal $n^{i}$ to the three-dimensional boundaries 
$B$ is defined to be outward pointing at the boundary $B_{+}$, inward pointing at the boundary $B_{-}$, and normalized so that $n \cdot n=+1$. In what follows we also assume that the foliation is restricted by the conditions $\left.(u \cdot n)\right|_{B}=0$.

In parallel to Ref. [7], the metric and extrinsic curvature of $\Sigma_{t}$ as a surface embedded in $M$ are denoted $h_{i j}$ and $K_{i j}=-h_{i}{ }^{k} \nabla_{k} u_{j}$ respectively, while the metric and extrinsic curvature of the boundaries $B$ as surfaces embedded in $M$ are $\gamma_{i j}$ and $\Theta_{i j}=-\gamma_{i}{ }^{k} \nabla_{k} n_{j}$. We denote by $h$ and $\gamma$ the determinants of the three-dimensional metrics $h_{i j}$ and $\gamma_{i j}$. The intersection of the boundaries $B_{+}$and $B_{-}$with $\Sigma_{t}$ are (topologically) spherical two-dimensional surfaces denoted by $S_{+}$and $S_{-}$respectively. The induced metric and extrinsic curvature of the boundaries $S$ as surfaces embedded on $\Sigma$ are $\sigma_{a b}$ and $k_{a b}=-\sigma_{a}{ }^{k} D_{k} n_{b}$ respectively. The normal vector $n^{i}$ to $B$ is also the normal vector to $S$. The simbols $\nabla$ and $D$ denote covariant differentiation with respect to the metrics $g_{\mu \nu}$ and $h_{i j}$ respectively. The metric tensors for the different surfaces can be written as

$$
\begin{aligned}
& h_{\mu \nu}=g_{\mu \nu}+u_{\mu} u_{\nu}, \\
& \gamma_{\mu \nu}=g_{\mu \nu}-n_{\mu} n_{\nu}, \\
& \sigma_{\mu \nu}=g_{\mu \nu}+u_{\mu} u_{\nu}-n_{\mu} n_{\nu} .
\end{aligned}
$$

In particular, we have

$$
\begin{aligned}
& \sqrt{-g}=|N| \sqrt{h}, \\
& \sqrt{-\gamma}=|N| \sqrt{\sigma}
\end{aligned}
$$

where $\sigma$ is the determinant of the two-dimensional metric $\sigma_{a b}$.

As shown in [7], the extrinsic curvature tensor $\Theta^{\mu \nu}$ can be written as

$$
\Theta^{\mu \nu}=k^{\mu \nu}+u^{\mu} u^{\nu} n_{\alpha} a^{\alpha}+2 \sigma^{\alpha(\mu} u^{\nu)} n^{\beta} K_{\alpha \beta}
$$

The traces of $\Theta_{\mu \nu}$ and $k_{\mu \nu}$ are related by

$$
\Theta=k-n_{\beta} a^{\beta}
$$


where $a^{\mu}=u^{\alpha} \nabla_{\alpha} u^{\mu}$ is the acceleration of the timelike normal $u^{\mu}$.

For the usual ("untilted") foliation the lapse function $N$ can be chosen everywhere positive, and there is no intersection between slices corresponding to different time parameter. In the case of a "tilted foliation", slices corresponding to different time parameter $t$ intersect at the two-dimensional bifurcation surface where the lapse function $N$ vanishes. We assume the parameter $N$ to be positive at $\Sigma_{+}$and negative at $\Sigma_{-}$.

\section{DYNAMICS OF A TILTED FOLIATION}

It is instructive to study the dynamics of "tilted" and "untilted" foliations independently. Consider at first the "tilted foliation" shown in Figure 3. The region lying between the two spacelike Cauchy surfaces $\Sigma^{\prime}$ and $\Sigma^{\prime \prime}$ consists of two wedges $M_{+}$and $M_{-}$intersecting at a two-dimensional surface $S_{0}$. The symbol $\Sigma_{t( \pm)}$ denotes the part of $\Sigma_{t}$ located in $M_{ \pm}$. We also denote those parts of $\Sigma^{\prime}$ and $\Sigma^{\prime \prime}$ which are the spacelike boundaries of the wedges $M_{ \pm}$ as $\Sigma_{ \pm}^{\prime}$ and $\Sigma_{ \pm}^{\prime \prime}$. The lapse function $N$ is positive (negative) at $M_{+}\left(M_{-}\right)$and equals zero at the bifurcation surface. The vector $u^{\mu}$ is future oriented in $M_{+}$and past oriented in $M_{-}$.

The covariant form of the gravitational action for this foliation with fixed three-geometry at the boundaries of $M$ is

$$
\begin{aligned}
S & =\frac{1}{2 \kappa} \int_{M_{+}} d^{4} x \sqrt{-g} \Re+\frac{1}{\kappa} \int_{(+) t^{\prime}}^{t^{\prime \prime}} d^{3} x \sqrt{h} K-\frac{1}{\kappa} \int_{B_{+}} d^{3} x \sqrt{-\gamma} \Theta \\
& -\frac{1}{2 \kappa} \int_{M_{-}} d^{4} x \sqrt{-g} \Re+\frac{1}{\kappa} \int_{(-) t^{\prime}}^{t^{\prime \prime}} d^{3} x \sqrt{h} K-\frac{1}{\kappa} \int_{B_{-}} d^{3} x \sqrt{-\gamma} \Theta,
\end{aligned}
$$

where $\Re$ denotes the four-dimensional scalar curvature and $\kappa \equiv 8 \pi$. It is assumed that the integrations are taken over the coordinates $x^{\mu}$ which have the same orientation as the canonical coordinates $(t, y, \theta, \phi)$ of the "tilted foliation". The negative sign for the integration over $M_{-}$reflects the fact that the canonical coordinates are left oriented in this region.

Besides the volume term, the action $S$ contains also boundary terms. The notation $\int_{( \pm) t^{\prime}}^{t^{\prime \prime}}$ represents an integral over the three-boundary $\Sigma_{ \pm}$at $t^{\prime \prime}$ minus an integral over the threeboundary $\Sigma_{ \pm}$at $t^{\prime}$. The action (3.1) has to be complemented [1. 10,13] with a subtraction 
term $S^{0}$ (dependent only on the boundary intrinsic geometry) which will be discussed later.

We write the Hamiltonian form of the action following the standard procedure by recognizing that there exists a direction of time at the boundaries inherited by the time vector field $t^{\mu}$ defined at $B$. Under a $3+1$ spacetime split, the four-dimensional scalar curvature is

$$
\Re=R+K_{\mu \nu} K^{\mu \nu}-(K)^{2}-2 \nabla_{\mu}\left(K u^{\mu}+a^{\mu}\right)
$$

where $R$ denotes the scalar curvature of the three-dimensional spacelike hypersurface $\Sigma$. By the use of Gauss' theorem, the conditions

$$
\left.u \cdot n\right|_{B}=0, u \cdot a=0, u \cdot u=-1, n \cdot n=1,
$$

and Eqns. (2.13)-(2.16) and (3.2), one can rewrite the total action in the form

$$
\begin{aligned}
S & =\frac{1}{2 \kappa} \int_{M_{+}} d^{4} x \sqrt{-g}\left[R+K_{\mu \nu} K^{\mu \nu}-(K)^{2}\right]-\frac{1}{\kappa} \int_{B_{+}} d^{3} x \sqrt{-\gamma} k \\
& -\frac{1}{2 \kappa} \int_{M_{-}} d^{4} x \sqrt{-g}\left[R+K_{\mu \nu} K^{\mu \nu}-(K)^{2}\right]-\frac{1}{\kappa} \int_{B_{-}} d^{3} x \sqrt{-\gamma} k,
\end{aligned}
$$

where $k=-D_{\mu} n^{\mu}$ on $B_{-}$. The regions $M_{+}$and $M_{-}$intersect at the cusp-like part $S_{0}$ of their boundaries. Generically such a cusp gives an additional contribution to the action [13]. For the "tilted foliation" the cusp contributions connected with the regions $M_{+}$and $M_{-}$have opposite signs. The regularity of the spacetime in the vicinity of $S_{0}$ implies that their absolute values are the same. That is why the action (3.4) does not contain any cusp contributions connected with $S_{0}$.

The momentum $P^{i j}$ conjugate to the three-metric $h_{i j}$ of $\Sigma$ is defined as

$$
P^{i j}=\frac{1}{2 \kappa} \sqrt{h}\left(K h^{i j}-K^{i j}\right)
$$

and the definition of $K_{\mu \nu}$ implies

$$
K_{i j}=-\nabla_{i} u_{j}=-\frac{1}{2 N}\left[\dot{h}_{i j}-2 D_{(i} V_{j)}\right],
$$

where the dot denotes differentiation with respect to the time $t$.

Observe that by virtue of (2.14), the kinetic part of the volume integral in (3.4) for both boundaries $B_{+}$and $B_{-}$can be written in the desired form 


$$
2 \kappa\left[P^{i j} \dot{h}_{i j}-2 P^{i j} D_{i} V_{j}-2 \kappa N G_{i j k \ell} P^{i j} P^{k \ell}\right]
$$

where $G_{i j k \ell}=\left(h_{i k} h_{j \ell}+h_{i \ell} h_{j k}-h_{i j} h_{k \ell}\right) /(2 \sqrt{h})$. The second term in the right hand side of (3.7) can be also rewritten since $-2 P^{i j} D_{i} V_{j}=-2\left[D_{i}\left(P^{i j} V_{j}\right)-V_{j} D_{i} P^{i j}\right]$ and

$$
-2 \int d^{4} x D_{i}\left(P^{i j} V_{j}\right)=-2 \int_{B_{+}} d^{3} x n_{i}\left(P^{i j} V_{j} / \sqrt{h}\right) \sqrt{\sigma}+2 \int_{B_{-}} d^{3} x n_{i}\left(P^{i j} V_{j} / \sqrt{h}\right) \sqrt{\sigma},
$$

with the cusp contributions at $S_{0}$ cancelling identically. Substitution of (3.7) into (3.4) gives us the desired Hamiltonian form of the action. In terms of the quantities [3,7]

$$
\varepsilon \equiv(k / \kappa), j^{i} \equiv-2 \sigma^{i} n_{\ell} P^{k \ell} / \sqrt{h}
$$

the action finally becomes

$$
S=\int_{M} d^{4} x P^{i j} \dot{h}_{i j}-\int H d t
$$

with the gravitational Hamiltonian $H$ given by

$$
H=\int_{\Sigma} d^{3} x\left(N \mathcal{H}+V^{i} \mathcal{H}_{i}\right)+\int_{S_{+}} d^{2} x \sqrt{\sigma}\left(N \varepsilon-V^{i} j_{i}\right)-\int_{S_{-}} d^{2} x \sqrt{\sigma}\left(N \varepsilon-V^{i} j_{i}\right)
$$

The Hamiltonian posseses both volume and boundary contributions. The volume part involves the gravitational contribution to the Hamiltonian and momentum constraints:

$$
\begin{aligned}
\mathcal{H} & =(2 \kappa) G_{i j k \ell} P^{i j} P^{k \ell}-\sqrt{h} R /(2 \kappa), \\
\mathcal{H}_{i} & =-2 D_{j} P_{i}{ }^{j}
\end{aligned}
$$

Notice that for stationary solutions, the first term of (3.10) is zero. If one imposes the gravitational constraint equations, the volume part of the Hamiltonian (3.11) is zero. The Hamiltonian can be written in the suggestive form

$$
H=H_{+}-H_{-},
$$

where the Hamiltonian $H_{+}$of $M_{+}$and the Hamiltonian $H_{-}$of $M_{-}$are

$$
\begin{aligned}
& H_{+}=\int_{S_{+}} d^{2} x \sqrt{\sigma}\left(N \varepsilon-V^{i} j_{i}\right) \\
& H_{-}=\int_{S_{-}} d^{2} x \sqrt{\sigma}\left(N \varepsilon-V^{i} j_{i}\right) .
\end{aligned}
$$




\section{QUASILOCAL ENERGY}

The quasilocal energy can be defined, following Ref. [0], as the value of the Hamiltonian that generates unit time translations orthogonal to the two-dimensional spacelike boundaries. The total quasilocal energy is therefore the value of the Hamiltonian (3.13) (with $\left.V_{i}=0\right)$ such that $|N|=1$ at both $S_{+}$and $S_{-}$, namely

$$
E_{\text {tot }}=E_{+}-E_{-}
$$

with

$$
\begin{aligned}
& E_{+}=\int_{S_{+}} d^{2} x \sqrt{\sigma}\left(\varepsilon-\varepsilon^{0}\right), \\
& E_{-}=-\int_{S_{-}} d^{2} x \sqrt{\sigma}\left(\varepsilon-\varepsilon^{0}\right) .
\end{aligned}
$$

This is the quasilocal energy of a spacelike hypersurface $\Sigma=\Sigma_{+} \cup \Sigma_{-}$bounded by two spacelike boundaries $S_{+}$and $S_{-}$located in the two regions $M_{+}$and $M_{-}$respectively. The quantity $\varepsilon$, defined in terms of the trace $k$ of extrinsic curvature in (3.9), is seen to be proportional to the surface energy density of the gravitational field. It is important to stress that for both boundaries $k=-D^{\mu} n_{\mu}$. The signs of $E_{+}$and $E_{-}$are directly connected with the signs of extrinsic curvatures of the surfaces $S_{+}$and $S_{-}$for the chosen orientation of the normal vector $n_{\mu}$. In the particular case of an eternal black hole, both the energies $E_{+}$and $E_{-}$are positive provided the surface $S_{+}$is chosen in the right wedge $M_{+}$and the surface $S_{-}$ is chosen in the left wedge $M_{-}$. The expressions (3.13)-(3.14) for the Hamiltonian as well as expressions (4.1)-(4.2) for the quasilocal energy are valid for any "tilted foliation".

We have included in these expression the relevant subtraction terms $\varepsilon^{0}$ for the energy [1.[7]. The role of the subtraction term is to normalize the action with the given boundary conditions to zero for a reference spacetime (usually taken to be flat spacetime). The reference space is a fixed hypersurface of this spacetime and $k^{0}$ the trace of extrinsic curvature in this space of a two-dimensional surface whose induced metric is $\sigma_{a b}$. I As indicated in

\footnotetext{
${ }^{1}$ The evaluation of this term is delicate, even for a single boundary surface, since there is no
} 
Eqn.(4.2) one needs to make the corresponding subtraction for each of the boundaries $B_{+}$ and $B_{-}$independently. As the result of this procedure, in the limiting case when $S_{ \pm}$tends to asymptotic spatial infinity the limiting values of either of the quantities $E_{+}$and $E_{-}$coincide with the ADM energy $\mathcal{M}$.

To illustrate this subtraction procedure and to gain some intuition about the relative signs of $E_{+}$and $E_{-}$, consider the following example, consisting of a static Einstein-Rosen bridge whose line element is:

$$
d s^{2}=-N^{2} d t^{2}+d y^{2}+r^{2}(y) d \Omega^{2}
$$

where $N$ and $r$ are functions of the proper distance coordinate $y$ continuously defined on $M$. The boundaries $S_{+}$and $S_{-}$are located at coordinate values $y=y_{+}$and $y=y_{-}$respectively. The normal to the boundaries is $n^{\mu}=\delta_{y}^{\mu}$. It is easy to see, by using the expression

$$
k=-\frac{1}{\sqrt{h}}\left(\sqrt{h} n^{\mu}\right)_{, \mu}
$$

that the trace $k$ at either boundary is

$$
k=\frac{-2 r_{, y}}{r}
$$

The value of $k$ depends on the function $r_{, y}$, which is positive for $S_{+}$and negative for $S_{-}$. The trace $k$ associated with the subtraction term is taken to be $k^{0}=-2 / r$ for $B_{+}$and $k^{0}=2 / r$ for $B_{-}$. This implies that the quasilocal energy (with subtraction terms included) can be written as

$$
\begin{aligned}
E & =E_{+}-E_{-} \\
& =\left(r\left[1-\left|r_{, y}\right|\right]\right)_{y=y_{+}}-\left(r\left[1-\left|r_{, y}\right|\right]\right)_{y=y_{-}} .
\end{aligned}
$$

The energies $E_{+}$and $E_{-}$have the same relative sign. The total energy is zero for boundary conditions symmetric with respect to the bifurcation surface $S_{0}$. The energy $E_{+}\left(E_{-}\right)$tends

definite prescription of how to to embed uniquely an arbitrary surface whose intrinsic metric is $\sigma_{a b}$ into a reference space. 
to the ADM mass $\mathcal{M}$ whenever the boundary $B_{+}\left(B_{-}\right)$tends to right (left) spatial infinity and the gravitational constraints are inforced. As an illustration of Eqn. (4.6), consider the case when the boundary $B_{+}$is located at right-hand infinity $\left(y_{+}=\infty\right)$ and the boundary $B_{-}$is located at $y_{-}$. The energy for this configuration is

$$
E=\mathcal{M}-r\left[1-\left(1-\frac{2 \mathcal{M}}{r}\right)^{1 / 2}\right],
$$

where $r=r\left(y_{-}\right)$. The energy is an even function of $y_{-}$(Figure 5). It is zero when $\left|y_{-}\right|=\infty$, and reaches a minimum value $(-\mathcal{M})$ at the bifurcation surface. The quasilocal energy is seen to be non-positive for all values of $y_{-}$when $B_{+}$is at right-hand infinity. This behavior is due to the fact that the quasilocal energy defined by (4.1)-(4.2) takes explicit account of gravitational binding.

The previous example illustrates a general property of the quasilocal energy (4.1): for a spacetime with a bifurcation surface, the quantities $E_{+}$and $E_{-}$have the same relative sign, while the total energy is given by the subtraction $E_{+}-E_{-}$[6]. The same property holds for the Hamiltonian. This is physically very attractive: the regions $M_{+}$and $M_{-}$of an eternal black hole are symmetric to each other, the time along the respective boundaries being determined by the global time $t$. The energies associated to $B_{+}$and $B_{-}$are symmetric and the total energy reflects the orientation reversal of the two boundaries. The dynamical aspects of the gravitational theory for spherically symmetric eternal black holes was recently considered by Kuchař [14]. Our expressions (3.14) reproduce his results when the black hole is spherically symmetric and both boundaries $B_{+}$and $B_{-}$are taken to their corresponding spatial infinities.

The time direction induced at the boundaries $B_{+}$and $B_{-}$considered above was inherited from the vector $t^{\mu}$. While the total action is independent of time orientations at the boundaries, the Hamiltonian does indeed depend on this orientation. 


\section{ANGULAR MOMENTUM}

The Hamiltonian form of the action given by (3.10) can be used to define charge-like quantities [7]. Consider in particular the definition of angular momentum. The value of the Hamiltonian function whenever $N=0$ at the boundaries $S_{+}$and $S_{-}$is $H=H_{+}-H_{-}$with

$$
\begin{aligned}
& H_{+}=-\int_{S_{+}} d^{2} x \sqrt{\sigma}\left(V^{i} j_{i}\right) \\
& H_{-}=-\int_{S_{-}} d^{2} x \sqrt{\sigma}\left(V^{i} j_{i}\right) .
\end{aligned}
$$

The momentum density $j^{i}$ of a spacelike hypersurface $\Sigma$, defined by Eqn. (3.9), is the value per unit area of the Hamiltonian that generates spatial diffeomorphisms in the $\partial / \partial x^{i}$ direction on the two-dimensional boundaries $S$. Assume that the boundary three-metrics admit a rotational symmetry represented by a Killing vector $\xi_{\phi}$. If $\xi_{\phi}$ is tangent to both $S_{+}$ and $S_{-}$(that is, both two-boundaries contain the orbits of $\xi_{\phi}$ ), the total angular momentum is defined as (minus) the value of the Hamiltonian that generates a unit rotation along $\xi_{\phi}$. That is, the total angular momentum is the value of $H$ with $N=0$ and $V^{i}=\xi_{\phi}{ }^{i}$ at the boundaries, namely

$$
J_{\text {tot }}=J_{+}-J_{-},
$$

with

$$
\begin{aligned}
& J_{+}=\int_{S_{+}} d^{2} x \sqrt{\sigma}\left(j_{i} \xi_{\phi}{ }^{i}\right), \\
& J_{-}=\int_{S_{-}} d^{2} x \sqrt{\sigma}\left(j_{i} \xi_{\phi}{ }^{i}\right) .
\end{aligned}
$$

As for the quasilocal energy, the angular momentum for the complete spacetime can be expressed as the subtraction of two terms of the same functional form. Each term is an integral over a two-surface (with unit normal orthogonal to $\xi_{\phi}$ ) of the momentum density in the $\xi_{\phi}$ direction. Notice in particular that for symmetric boundary conditions the total angular momentum is zero. 


\section{DYNAMICS OF UNTILTED FOLIATIONS}

Consider the case of an "untilted" foliation illustrated in Figure 4. This foliation is regular everywhere in a region of $M$ lying between the initial $\Sigma^{\prime}$ and final $\Sigma^{\prime \prime}$ slices. The spacetime is spatially bounded by the timelike boundaries $B_{-}$and $B_{+}$. Let $t$ denote the global time parameter labeling the slices $\Sigma$. The coordinates $(y, \theta, \phi)$ are defined as in previous sections. The foliation is such that $u^{\mu}=-N \delta^{\mu}$, with the lapse function defined everywhere positive. For simplicity, we consider only the special case $V^{i}=0$. The gravitational action when the three-geometry is fixed at the boundaries takes the form:

$$
\begin{aligned}
S=\frac{1}{2 \kappa} \int_{M} d^{4} x \sqrt{-g} \Re & +\frac{1}{\kappa} \int_{(+) t^{\prime}}^{t^{\prime \prime}} d^{3} x \sqrt{h} K-\frac{1}{\kappa} \int_{B_{+}} d^{3} x \sqrt{-\gamma} \Theta \\
& -\frac{1}{\kappa} \int_{(-) t^{\prime}}^{t^{\prime \prime}} d^{3} x \sqrt{h} K+\frac{1}{\kappa} \int_{B_{-}} d^{3} x \sqrt{-\gamma} \Theta-S^{0},
\end{aligned}
$$

where $S^{0}$ represents the subtraction action. By using the general decomposition (3.6), the action in Hamiltonian form becomes

$$
S=\int_{M} d^{4} x\left[P^{i j} \dot{h}_{i j}-N \mathcal{H}\right]-\int_{B_{+}} d^{3} x \sqrt{\sigma} N \varepsilon+\int_{B_{-}} d^{3} x \sqrt{\sigma} N \varepsilon-S^{0}
$$

The boundary Hamiltonian is now

$$
H=\int_{S_{+}} d^{2} x \sqrt{\sigma} N\left(\varepsilon-\varepsilon^{0}\right)-\int_{S_{-}} d^{2} x \sqrt{\sigma} N\left(\varepsilon-\varepsilon^{0}\right)
$$

and the quasilocal energy is

$$
\begin{aligned}
& E_{\mathrm{tot}}=E_{+}-E_{-}, \\
& E_{+}=\int_{S_{+}} d^{2} x \sqrt{\sigma}\left(\varepsilon-\varepsilon^{0}\right), \\
& E_{-}=\int_{S_{-}} d^{2} x \sqrt{\sigma}\left(\varepsilon-\varepsilon^{0}\right) .
\end{aligned}
$$

The expression (6.3) for the Hamiltonian as well as the expressions (6.4) for the quasilocal energy are valid for any "untilted" foliation. The signs of $E_{+}$and $E_{-}$are directly connected with the signs of extrinsic curvatures of the surfaces $S_{+}$and $S_{-}$for the chosen direction of the normal $n^{\mu}$. Expressions (6.4) are to be contrasted with Eqns.(4.1)-(4.2) for a "tilted foliation". 
The same results can be obtained from a covariant analysis. Consider for simplicity a spherically symmetric spacetime. The normal vector $n$, defined at the boundary, can be continued to the interior of $M$ by assuming that $n \cdot \nabla y$ is positive throughout. The scalar curvature can be written as [2]

$$
\Re=\frac{-2}{\sqrt{-g}}\left(\sqrt{-\gamma} n^{\alpha} a_{\alpha}\right)_{, y}-2 G_{0}^{0} .
$$

The action is easily obtained by applying Gauss' theorem to the first term of (6.5). By enforcing the Hamiltonian constraint $G^{0}{ }_{0}=0$, and using Eqn. (2.16) for the trace of extrinsic curvature, the action reduces to the boundary action:

$$
S_{B}=-\int_{B_{+}} d^{3} x \sqrt{\sigma} N \varepsilon+\int_{B_{-}} d^{3} x \sqrt{\sigma} N \varepsilon-S^{0},
$$

which agrees with the action (6.2) when the gravitational constraints and the staticity conditions are imposed.

Consider as a particular example a static, spherically symmetric eternal black hole generated by an untilted foliation when both boundaries $B_{+}$and $B_{-}$are located in the 'right' wedge $M_{+}$. For the natural choice of global time $t$ as the parameter numerating the slices, one obtains the quasilocal energy

$$
E=\left(r\left[1-\left|r_{, y}\right|\right]\right)_{y=y_{2}}-\left(r\left[1-\left|r_{, y}\right|\right]\right)_{y=y_{1}} .
$$

where $y=y_{ \pm}$denotes the location of the boundaries $B_{ \pm}$respectively. Both energies $E_{+}$ and $E_{-}$are positive when the surfaces $S_{+}$and $S_{-}$are in the 'right' wedge $M_{+}$. It can be seen that this energy is of the same form as (4.6). This means that the quasilocal energy expressions for an eternal black hole are of the same form whether the 'left' boundary is located in $M_{+}$or in $M_{-}$, provided the global time $t$ is chosen as the natural scalar function labeling the foliation (that is, provided the foliation is "untilted" when the 'left' boundary lies in $M_{+}$and 'tilted" when the 'left' boundary lies in $M_{-}$).

The expressions (6.4) for the quasilocal energy of an "untilted foliation" formally coincide with the quasilocal energy (4.1)-(4.2) for "tilted foliations" if one formally changes the 
direction of time $t$ at one of the boundaries (that is, if one formally changes the direction of time in $B_{-}$to be $t_{-}=-t_{+}$, where $t=t_{+}$denotes the time direction in $B_{+}$and in the interior of $M)$.

\section{CONCLUDING REMARKS}

We discuss now a possible application of the eternal black hole Hamiltonian (3.13)-(3.14) to the thermofield dynamics approach to quantum processes in black holes. Thermofield dynamics is a theory of thermal fields based on the idea of augmenting the physical Fock space $\mathcal{F}$ by a fictitious Fock space $\tilde{\mathcal{F}}$ obtained from the first one by a conjugation operation [9]. This results in a doubling of degrees of freedom for the system. A temperature dependent "vacuum state" can be defined on the total Fock space $\mathcal{F} \otimes \tilde{\mathcal{F}}$ in such a way that vacuum expectation values of any physical operator agree with their statistical average for an ensemble in thermal equilibrium. A pure state in $\mathcal{F} \otimes \tilde{\mathcal{F}}$ will then correspond to a mixed state in $\mathcal{F}$. This approach is self-consistent if the total Hamiltonian for this extended system is of the general form

$$
H=H_{+}-H_{-},
$$

where $H_{+}$is the Hamiltonian associated to $\mathcal{F}$ and $H_{-}$the Hamiltonian associated to $\tilde{\mathcal{F}}$.

In the original formulation of thermofield dynamics the space $\tilde{\mathcal{F}}$ is merely formal. However, Israel [8] has suggested that a thermofield dynamics approach may give an adequate description of black hole radiation and explain its thermal nature. An 'eternal' version of a black hole contains two causally disconnected regions $R_{+}$and $R_{-}$. The states of Boulware particles in $R_{+}$and $R_{-}$can be identified with the Hilbert spaces $\mathcal{F}$ and $\tilde{\mathcal{F}}$ respectively, so

that the space $\mathcal{F} \otimes \tilde{\mathcal{F}}$ describes states of the complete system. Israel's suggestion is the result of an explicit calculation that shows that an observer in the right wedge $R_{+}$observes particle modes travelling in the background of a spacetime with a horizon as having thermal behavior [8]. (For further development of this idea, see for example Refs. [15].) This analysis 
is based only on the study of small perturbations propagating in a black hole background. It is interesting to note that our general result for the Hamiltonian for the "tilted foliation" indicates that in the general case (even in one does not restrict oneself by considering only small perturbations) the complete Hamiltonian (3.13) of the system has the same structure as the Hamiltonian (7.1), provided that the timelike surfaces bounding the system are located in different wedges. The Hamiltonians $H_{+}$and $H_{-}$can be used for the definition of vacuum states and for the construction of two Fock spaces. We have seen that the Hamiltonians $H_{+}$and $H_{-}$are encoded in the boundary conditions. In a special case when the boundary conditions on $B_{+}$and $B_{-}$are the same, $H_{+}$and $H_{-}$are identical. The property of the Hamiltonian mentioned above indicates that the thermofield dynamics approach to black hole thermodynamics developed by Israel for small perturbations might be naturally generalized to the situation when the perturbations are not assumed to be small.

The description of dynamics of an eternal black hole requires data on two timelike boundary surfaces $B_{+}$and $B_{-}$. It is natural to relate the data on $B_{+}$and $B_{-}$with the external and internal degrees of freedom of a black hole respectively. This opens the possibility of generalizing the path integral approach to black hole thermodynamics to the case when the internal degrees of freedom of the black hole are present. It would be interesting to develop this analysis and apply it to the problem of dynamical origin of black hole entropy.

\section{ACKNOWLEDGMENTS}

The authors have benefitted from discussions with Andrei Barvinsky, Geoff Hayward, and Andrei Zelnikov. They would also like to thank Werner Israel for his interest and useful remarks. Reseach support was received from the Natural Sciences and Engineering Research Council of Canada. 


\section{REFERENCES}

[1] G. W. Gibbons and S. W. Hawking, Phys. Rev. D15, 2752 (1977); S. W. Hawking, in General Relativity, edited by S. W. Hawking and W. Israel (Cambridge University Press, Cambridge, 1979).

[2] J. W. York, Jr., Phys. Rev. D 33, 2092 (1986); B. F. Whiting and J. W. York, Jr., Phys. Rev. Lett., 61, 1336 (1988).

[3] J. D. Brown, E. A. Martinez, and J. W. York, Jr., Phys. Rev. Lett., 66, 2281 (1991); in Nonlinear Problems in Relativity and Cosmology, edited by J. R. Buchler, S. L. Detweiler, and J.R. Ipser (New York Academy of Sciences, New York, 1991).

[4] A. O. Barvinsky, V. Frolov, and A. Zelnikov, preprint, Alberta-Thy-13-94, grqc/9404036, (1994).

[5] J. D. Brown and J. W. York, Jr., Phys. Rev. D 47, 1420 (1993).

[6] V. Frolov and E. A. Martinez, "Eternal black holes and quasilocal energy", AlbertaThy-19-94, gr-qc/9405041, to appear in Proceedings of the Lake Louise Winter Institute on Particle Physics and Cosmology, World Scientific, 1994.

[7] J. D. Brown and J. W. York, Jr., Phys. Rev. D 47, 1407 (1993).

[8] W. Israel, Phys. Lett. 57A, 107 (1976).

[9] H. Umezawa and Y. Takahashi, Collective Phenomena 2, 55 (1975); H. Umezawa, Advanced Field Theory, American Institute of Physics, New York, 1993.

[10] C. W. Misner, K. S. Thorne and J. A. Wheeler, Gravitation, W. H. Freeman, San Francisco, 1973.

[11] R. Arnowitt, S. Deser, and C. W. Misner, in Gravitation: An Introduction to Current Research, edited by L. Witten (Wiley, New York, 1962). 
[12] J. M. Bardeen, in Black Holes, C. DeWitt and B. S. DeWitt, eds., Gordon and Breach Science Publishers, New York, 1973.

[13] D. Brill and G. Hayward, preprint UMD-PP 94-100, Alberta-Thy-10,94, gr-qc/9403018, (1994).

[14] K. V. Kuchař, preprint, UU-REL-94/3/1, gr-qc/9403003, (1994).

[15] R. Laflamme, Nucl. Phys. B324, 233 (1989); N. Sanchez and B. F. Whiting, Nucl. Phys. B283, 605 (1987); B. F. Whiting, in Proceedings of the Workshop on Thermal Field Theories and their applications, edited by K. L. Kowalski, N. P. Landsman, and Ch. G. van Weert, Physica A, North Holland (1988). 


\section{FIGURE CAPTIONS}

Figure 1: Penrose diagram for a static, spherically symmetric spacetime. Each point in the diagram represents a topological sphere $S^{2}$. The bifurcation surface is denoted by $S_{0}$. The spacetime is the union of four regions $R_{ \pm}$and $T_{ \pm}$ separated by the future and past horizons $H^{ \pm} . B_{+}$and $B_{-}$represent two timelike surfaces located in the regions $R_{+}$and $R_{-}$respectively. The dashed lines in the figure represent 'initial' and 'final' spacelike hypersurfaces $\Sigma$ ' and $\Sigma$ " of a "tilted foliation". (For simplicity, tildes have been omitted.)

Figure 2: A slice $\Sigma$ (Einstein-Rosen bridge) of a static, spherically symmetric eternal black hole. The surface $\Sigma$ has wormhole topology $R^{1} \times S^{2}$. The parts of $\Sigma$ lying in $R_{ \pm}$are $\Sigma_{ \pm}$respectively, and $\Sigma=\Sigma_{+} \cup \Sigma_{-}$. The timelike boundaries $B_{+}$and $B_{-}$cross the Einstein-Rosen bridge at two-dimensional surfaces $S_{+}$and $S_{-}$. The bifurcation surface is denoted by $S_{0}$. (Tildes have been omitted for simplicity.)

Figure 3: A "tilted foliation" $\Sigma$ of $M$ extending between the initial $\left(\Sigma^{\prime}\right)$ and final $\left(\Sigma^{\prime \prime}\right)$ spacelike hypersurfaces. Each point in the diagram is a topological sphere $S^{2}$. All the slices in the foliation intersect at the bifurcation surface $S_{0}$. The timelike boundaries $B_{ \pm}$intersect a spacelike surface $\Sigma$ at the two disconnected two-dimensional surfaces $S_{ \pm}$.

Figure 4: An "untilted foliation" of $M$ extending between the hypersurfaces $\Sigma^{\prime}$ and $\Sigma^{\prime \prime}$. Slices corresponding to different time parameters do not intersect.

Figure 5: The quasilocal energy $E$ for a static, spherically symmetric Einstein-Rosen bridge when the right boundary $S_{+}$is located at spatial infinity. The location of the left boundary $S_{-}$is denoted by $y_{-}$. The horizon corresponds to $y_{-}=0$. 
This figure "fig1-1.png" is available in "png" format from: http://arxiv.org/ps/gr-qc/9411001v1 
This figure "fig1-2.png" is available in "png" format from: http://arxiv.org/ps/gr-qc/9411001v1 
This figure "fig1-3.png" is available in "png" format from: http://arxiv.org/ps/gr-qc/9411001v1 
This figure "fig1-4.png" is available in "png" format from: http://arxiv.org/ps/gr-qc/9411001v1 
This figure "fig1-5.png" is available in "png" format from: http://arxiv.org/ps/gr-qc/9411001v1 